\section{Language maths}

Formal Languages. By Arto Salomaa. (ACM Monograph Series.) Pp. xiii+ 322. (Academic (Harcourt Brace Jovanovich): New York and London, May 1973.) $\$ 19$.

THIs excellent book is unique in its scope, detail and elegance. It covers almost the whole of formal language theory up to early 1972 in more or less detail, always with great clarity of style. The emphasis throughout is on pure mathematical theory-there is almost no discussion of applications. The author assumes a considerable mathematical stamina in his readers, and although the rigour of the text is considerably alleviated by many examples and side remarks, I would not recommend it as a first introduction to the field for most undergraduates.

The book is divided into three parts. The first (about a third of the book) is a very complete account of the classical theory of the basic hierarchy. The emphasis throughout is on the characterisation of language families by different means and the effects of various operations upon language families. Concepts of recursion theory are introduced informally at first but, in chapter 3, Smallyan's "rudimentarypredicate" characterisation is introduced as a descriptive device for type $\mathrm{O}$ languages. There is considerable new material, notably workspace theory. This section would form an excellent nucleus text for a short graduate course in formal language theory.

The second part-about half the book -is its real raison d'etre for the professional reader. It amounts to a connected self-contained survey of all major and several minor developments in modern formal language theory. Topics covered-AFLs, controlled grammars of various kinds; Lindenmayer systems (very interesting); a very full discussion of context-free languages, including power series analysis, LR(k) and LL(k) grammars and ambiguity; and finally a sort of linguistic dustbin containing transformational, categorical, indexed, scattered context and probabilistic grammars. Some of these are treated rather briefly, but references to other fuller discussions are given. (Brief bibliographical and historical remarks follow each chapter.) The scope of this section, the detail and rigour of the proofs, and the pervading sense of mathematical elegance, make these chapters a unique reference source for the field. The author gives many fascinating side remarks and illustrative examples.

The third part seems like an appendage. It consists of a fairly standard account of decidability and a nice introduction to complexity theory. The former would have been better placed nearer the beginning of the book: the latter should either have been omitted altogether, or should have been expanded to include (for example) a discussion of polynomial-time results for nondeterministic recognisers. At present it is an uneasy compromise.

The author has tried to give a complete account of a large subject. One can question whether it was sensible to make the attempt at this time, when the field is expanding so rapidly. Perhaps the publishers could leave a few blank pages in the next edition so that readers could jot down new results as they appear.

The book is technically well produced. I could find no significant misprints. Patrick J. Hayes

\section{Brain damage}

The Working Brain: An Introduction to Neuropsychology. By A. R. Luria. Translated by Basil Haigh. Pp. 398. (Allen Lane: London; Penguin: Harmondsworth, April 1973.) £2.50; Penguin $£ 1.25$.

ONE of the main problems with physiological psychology or neuropsychology is the crudity of its techniques relative to the complexity and subtlety exhibited by its subject matter, the brain. In this book one of the world's most eminent neuropsychologists surveys his vast work on the psychological effects of what is perhaps the crudest technique, brain damage. His purpose is to substantiate the claim that the resulting dysfunctions can tell one much about the functional structure underlying the $\operatorname{cog}$ nitive and perceptual properties of the human mind and the mechanisms by which they are manifest in overt behaviour.

In the opening part of the book, Luria discusses lesion methodology and rejects the simple 'localisation of function' procedure in which the dysfunction is described as the loss of a certain ability; a process to mediate that ability is specified, and finally this process is localised in the particular brain area damaged. It is clear that in a complex mechanism such as the brain there is not necessarily any simple mapping between the domains of functional and anatomical organisation. The outcome of brain damage, defined by anatomical criteria, could well be behavioural disturbance which does not reflect in any meaningful way the structure of the processes mediating normal functioning. Luria's answer to this problem is that a "detailed psychological analysis of the structure of the disturbance and the elucidation of the immediate causes of the collapse of the functional system" is necessary. But here is the paradox. If an adequate psychological theory of a given ability is a necessary prerequisite of a "detailed qualification of the symptom", what additional information about the psychological organisation can the study of brain damage give us? The question is whether the rest of the book provides an adequate answer.

Luria divides the brain into three principal functional units: the reticular activating system and the limbic system for regulating the arousal of the cortex and controlling emotional and motivational processes, the primary and seconJary projection areas and nonspecific areas of the posterior cortex for processing and storage of sensory information, and finally the motor and premotor areas and frontal lobes for programming, regulation, and verification of activity. In the second part, the author summarises a large body of evidence, mainly from his own or associated sources, on the effects of brain damage in various cortical areas. This material is then recapitulated in the third part and organised in terms of psychological processes rather than anatomical criteria.

Although a summary of Luria's extensive work must be of value, the relatively naive reader has difficulty in evaluating the adequacy with which the dysfunctions are presented. Most of the descriptions are supported solely by clinical evidence and rarely are controldata given for comparison. This difficulty is compounded by the appalling and often incomprehensible presentation of the figures; graphs have unlabelled axes and examples have unexplained legends. The theoretical versus descriptive status of the terms in which a syndrome is presented is often ambiguous, and I get the impression that Luria, in spite of his caution against 'the localisation of function' procedure, often reifies deficits. Luria's theoretical position is eclectic and he draws on many sources in specifying the psychological processes he thinks underlie the capacities of the mind. But the general theoretical mechanisms are presented in such an unspecified form that it is very difficult to decide whether brain damage really tells us anything new about psychological processes. A conclusion, which could find supportive evidence in this book, is that psychological theory is still too primitive to allow the proper use of the patterns of behavioural breakdown induced by brain damage.

\section{ANTHONY DICKINSON}

\section{New book?}

Quantum Statistical Properties of Radiation. By W. H. Louisell. Pp. xiv +528 . (Wiley: New York and London, July 1973.) £14.

THIs book is really a second edition of the author's 1964 volume Radiation and Noise in Quantum Electronics published by McGraw-Hill. Why he has changed his publisher and title I cannot 